\title{
El Yoga como método cuerpo-mente para mujeres embarazadas con depresión: una revisión sistemática
}

\author{
Julián NIETO SÁNCHEZ ${ }^{1}$, Antonio GRANERO-GALLEGOS ${ }^{1,2}$ \\ y María CARRASCO-POYATOS ${ }^{1,2}$ \\ ${ }^{1}$ Facultad de Ciencias de la Educación, Universidad de Almería, España \\ ${ }^{2}$ Centro de Investigación en Salud, Universidad de Almería, España \\ (Recibido el 28 de Junio de 2019, Aceptado el 3 de Noviembre de 2019)
}

\section{RESUMEN:}

Introducción: La Organización Mundial de la Salud considera la depresión prenatal como un gran problema de salud pública que actualmente afecta entre el 10-20\% de las mujeres gestantes, debido principalmente a la gran liberación de la hormona cortisol durante el embarazo. En los últimos años, algunas disciplinas como el Yoga, considerado como un método cuerpo-mente formado por técnicas de respiración, posturas físicas (Asanas) y meditación/relajación, ha ganado importancia como terapia alternativa contra la depresión prenatal por su relación con los índices y los síntomas de ansiedad o estrés en mujeres gestantes.

Objetivo: realizar una revisión sistemática de estudios controlados y aleatorizados sobre los cambios que genera el Yoga en el estado de depresión de mujeres embarazadas.

Método: Siguiendo la Declaración Prisma, se buscó en las siguientes bases de datos: Pubmed, Scopus, Medline, Current Contents Connect, Ebsco, Web of Science y CINAHL-Complete artículos publicados en los últimos 10 años y teniendo en cuenta unos criterios de inclusión y exclusión específicos. La estrategia de búsqueda fue: "(Yoga) AND (Pregnant OR Prenatal OR Antenatal) AND (Depression)". Se seleccionaron siete trabajos con calidad metodológica $\geq 6$ según la escala PEDro.

Resultados: Los artículos que obtuvieron resultados favorables $(n=4)$ presentaban: alta participación ( $\mathrm{n}>90$ sujetos), buen periodo de seguimiento de los sujetos ( $\mathrm{n} \geq 12$ semanas) y alta calidad metodológica ( $\mathrm{n} \geq 7$ puntos). Los artículos con resultados desfavorables $(\mathrm{n}=3)$ presentaban: baja participación $(\mathrm{n}<60$ sujetos) y escaso periodo de seguimiento ( $\mathrm{n} \leq 9$ semanas).

Conclusión: El Yoga ha resultado ser una terapia alternativa favorable para reducir los índices de depresión en mujeres gestantes.

Palabras clave: Ansiedad, estrés, cortisol, depresión, bienestar, prenatal

Yoga as a body-mind method for pregnant women with depression: a systematic review

\begin{abstract}
:
Introduction: The World Health Organization considers prenatal depression as a major public health problem that currently affects $10-20 \%$ of pregnant women. It is mainly due to the great release of the (c) Psy, Soc, \& Educ, 2020, Vol. 12(1)
\end{abstract}


hormone cortisol during pregnancy. In recent years, some disciplines such as Yoga, as a body-mind method including breathing techniques, physical postures (asanas) and meditation / relaxation, have gained importance as an alternative therapy against prenatal depression due to its relationship with the indexes and the symptoms of anxiety or stress in pregnant women.

Objective: carry out a systematic review of randomized controlled studies on the changes that Yoga generates in the state of depression of pregnant women.

Method: Following the Prisma Declaration, the search was conducted in the following databases: Pubmed, Scopus, Medline, Current Contents Connect, Ebsco, Web of Science and CINAHL-Complete. The articles included should be published in the last 10 years and should accomplish some inclusion and exclusion criteria. The search strategy was: "(Yoga) AND (Pregnant OR Prenatal OR Antenatal) AND (Depression)". Seven works with methodological quality $\geq 6$ according to the PEDro scale were selected.

Results: The articles that obtained favorable results $(n=4)$ presented: high participation $(n>90$ subjects), good period of follow-up of the subjects ( $\mathrm{n} \geq 12$ weeks) and high methodological quality ( $\mathrm{n} \geq 7$ points). The articles with unfavorable results $(n=3)$ presented: low participation $(\mathrm{n}<60$ subjects $)$ and short follow-up period ( $\mathrm{n} \leq 9$ weeks).

Conclusion: Yoga has turned out to be a favorable alternative therapy to reduce depression rates in pregnant women.

Keywords: Anxiety, stress, cortisol, depression, wellness, prenatal

Correspondencia: María Carrasco-Poyatos. Facultad de Ciencias de la Educación, Universidad de Almería. Edificio CITE-III, despacho 201. Ctra. De Sacramento s/n, 04120, La Cañada de San Urbano Almería. Teléfono: +34 950 214773. E-mail: carrasco@ual.es

\section{Introducción}

El embarazo es un periodo de estrés fisiológico que requiere que la gestante realice adaptaciones a nivel físico, mental y social, debido a los cambios que se producen en estas tres áreas (Satyapriya, Nagarathna, Padmalatha, \& Nagendra, 2013). Este estado de estrés, sumado a posibles situaciones externas estresantes (e.g., muerte de un ser querido, enfermedad familiar, divorcio, carga de trabajo, pérdida de empleo) pueden conducir a resultados prenatales adversos (Obel et al., 2005), como la depresión prenatal (Ngai \& Chan, 2011) o la depresión posparto (Heron et al., 2004). Los estudios han demostrado los problemas que acarrea padecer estrés materno prenatal, encontrando entre ellos un mayor riesgo de aborto espontáneo, parto prematuro, malformaciones fetales, y retraso del crecimiento asimétrico (Mulder et al., 2002). La respuesta del cuerpo ante el estrés mencionado está controlada por el eje hipotálamopituitaria-suprarrenal (HPA), dónde el hipotálamo produce un factor liberador de la hormona corticotropina que a su vez estimula la pituitaria a producir adrenocorticotropina, conduciendo a la secreción suprarrenal de cortisol (Chida \& Steptoe, 2009), proceso que sumado a la liberación de cortisol suprarrenal por parte de la placenta (O’Donnell, O'Connor, \& Glover, 2009), permite que esta hormona se encuentre durante el embarazo en dosis elevadas en el organismo, situación que se relaciona con la depresión en el embarazo.

La depresión está actualmente considerada un problema de salud pública con gran prevalencia mundial (Davis, Goodman, Leiferman, Taylor, \& Dimidjian, 2015), siendo catalogada por la Organización Mundial de la Salud (OMS) como el mayor contribuyente a la 
discapacidad global en 2015 (WHO, 2017). Asimismo, según Belay, Moges, Hiksa, Arado, \& Liben (2018), la depresión prenatal queda definida como la aparición de un proceso depresivo durante el embarazo y que afecta al $10-20 \%$ de las mujeres gestantes a nivel mundial. Las mujeres con depresión prenatal presentan un aumento del riesgo de padecer depresión posparto, insatisfacción conyugal, escaso apoyo social, posteriores problemas de estrés parental (Goodman \& Tully, 2009; Misri et al., 2010), prematuridad del parto (Field et al., 2009), retrasos del desarrollo (Deave, Heronm, Evans, \& Edmond, 2008) y, además, según de Bruijn, van Bakel, y van Baar (2009), sus futuros hijos tendrán mayor posibilidad de sufrir problemas de conducta tanto en su infancia, como en su etapa adolescente (Hay, Pawlby, Waters, Perra, \& Sharp, 2010). A pesar de lo expuesto, la mayoría de las mujeres embarazadas, siendo conocedoras de lo que estos problemas pueden causar en el feto y en ellas mismas, se muestran reacias a recibir atención de salud mental (Byatt, Simas, Lundquist, Johnson, \& Ziedonis, 2012).

Las clases de preparación para el parto buscan educar a la mujer gestante y su pareja sobre aspectos como la atención médica prenatal, el parto, la nutrición, el ejercicio preparto y postparto o la adecuada técnica de lactancia (Sturrock \& Johnson, 1990). Además, reporta una serie de beneficios, entre ellos, aumentar la autoconfianza de las mujeres antes del parto (Enkin et al., 2000) y mejorar el estado psicológico de las mujeres embarazas (Aznal, Wong, Tan, See, \& Wong, 2014).

Los tratamientos clásicos como los antidepresivos o las psicoterapias han sido infrautilizados en los casos de depresión prenatal debido a las contraindicaciones existentes sobre el feto (Einarson, Choi, Einarson, \& Koren, 2010). Este hecho se apoya en artículos de revisión que afirman que no existen estudios controlados sobre la seguridad de tomar antidepresivos durante el embarazado y la lactancia (Mian, 2005), mientras que, en el caso de las psicoterapias, éstas se tornan inaccesibles para la mayoría de mujeres (Parker, Crawford, \& Hadzi-Pavlovic, 2008). Las mujeres embarazadas optan preferentemente por los tratamientos no farmacológicos (Goodman, 2009), por lo que se podría abogar por tratamientos alternativos que no necesitasen de farmacología, como el ejercicio y la actividad física, que pueden prevenir la aparición de depresión al incidir en los niveles de neurotransmisores y hormonas ligados con la misma (Szegda et al., 2018).

En este sentido, el Yoga es una disciplina cuerpo-mente formada por técnicas de respiración, posturas físicas (Asanas) y meditación/relajación, que promueven la salud, así como también aumentan el bienestar espiritual de quienes lo practican (Chandler, 2001). El Yoga prenatal difiere de lo mencionado previamente, en el ritmo, la elección de las posturas, el uso de modificaciones y el énfasis en los cambios físicos del embarazo (Rakhshani et al., 2012). Se ha documentado que las mujeres que practican Yoga prenatal pueden experimentar beneficios que conllevan mejores resultados en el momento del parto (Rakhshani et al., 2012), incluyendo menos dolor, así como menos duración del mismo (Chuntharapat, Petpichetchian, \& Hatthakit, 2008). Además, la práctica del Yoga reporta efectos positivos como disminución del estrés y la ansiedad (Beddoe, Yang, Kennedy, Weiss, \& Lee, 2009), y de molestias y de dolor durante el embarazo (Beddoe et al., 2009; Sun, Hung, Chang, \& Kuo, 2010), por lo que el Yoga se muestra como una alternativa viable y segura a los clásicos tratamientos 
farmacológicos y que puede reducir la sintomatología que la depresión produce en las gestantes (Battle, Uebelacker, Magee, Sutton, \& Miller, 2015).

A partir de lo expuesto, se hace necesaria una revisión de estudios que contemple investigaciones relacionadas con las ideas expuestas, por lo que el presente trabajo tuvo como objetivo realizar una revisión sistemática de estudios controlados y aleatorizados sobre los cambios que genera el Yoga en el estado de depresión de mujeres embarazadas.

\section{Método}

La revisión sistemática (RS) se ha realizado siguiendo las directrices planteadas en la normativa PRISMA (Urrutia \& Bonfill, 2010), y se han incluido aquellos estudios que presentan como núcleo de estudio al Yoga y los efectos que éste produce en embarazadas con depresión. Para el estudio no hubo registro previo de protocolo de revisión.

Para la selección de los trabajos se siguieron los siguientes criterios:

a) Criterios de inclusión:

a. Ensayos Clínicos Aleatorizados (RCT, por sus siglas en inglés).

b. Publicaciones en inglés y español entre enero de 2009 y enero de 2019.

c. Uso del Yoga como terapia contra la depresión en mujeres embarazadas.

d. Efectos del Yoga en otras patologías relacionadas con la salud mental (e.g., ansiedad, estrés).

e. Tipos de intervenciones: Yoga vs intervención inespecífica, Yoga vs otra intervención activa, Yoga + intervención activa vs intervención activa sin Yoga, Yoga vs no intervención.

b) Criterios de exclusión

a. Estudios cuasi-experimentales sin aleatorización muestral.

b. Uso de fármacos antidepresivos que puedan interferir en el efecto del Yoga.

c. Estudios con calidad metodológica baja según escala PEDro.

Los estudios científicos publicados fueron identificados exclusivamente a través de búsquedas bibliográficas en las siguientes bases de datos electrónicas: Pubmed, Scopus, Web of Sciences (WOS), Medline, Current Contents Connect, Ebsco y CINAHL-Complete. La plataforma virtual de la Biblioteca de la Universidad de Almería fue la elegida para acceder a las diferentes bases de datos, con fecha de inicio de la búsqueda el 1 de enero de 2019, y posteriores modificaciones hasta abril del mismo año. Los términos de búsqueda utilizados se basaron en el Tesauro de Pubmed (MeSH): "Yoga", combinado con los términos relacionados con los periodos del embarazo, "Prenatal", "Antenatal, "Pregnant", y el término relacionado con los resultados a obtener "Depression". Los descriptores elegidos para la realización de la búsqueda, tras la combinación de los mismos fueron los siguientes: "(Yoga) AND (Pregnant OR Prenatal OR Antenatal) AND (Depression)".

Se realizaron tres cribados para la selección final de los artículos. Tanto la búsqueda como la selección de los artículos y su cribado fueron realizadas por dos evaluadores de forma independiente. Los desacuerdos fueron resueltos por consenso. La recopilación de datos se realizó en una hoja Excel (Microsoft Excel 2018 para Windows), extrayendo los siguientes datos: métodos, participantes, intervención, resultados, notas, sesgos o calidad metodológica. 
En cuanto a la evaluación de la calidad metodológica (riesgo de sesgo) de los artículos, se ha utilizado la escala PEDro, considerando únicamente los artículos con alta calidad metodológica según Elkins, Herbert, Moseley, Sherrington, y Maher (2010), y siguiendo el método establecido por Van Tulder, Furlan, Bombardier, y Bouter (2003).

\section{Resultados}

\section{Selección de estudios}

Conforme a los criterios de elegibilidad establecidos, la búsqueda inicial reportó un total de 282 artículos. Tras eliminar los duplicados, los artículos que no cumplían los criterios especificados, y aquellos con menos de 6 puntos de calidad metodológica, quedaron un total de 7 artículos que fueron evaluados e incluidos en el presente trabajo. En la Figura 1 se expone el diagrama de flujo de las distintas fases que los artículos debieron superar para estar incluidos en la RS.

\section{Características de los estudios}

Los estudios y sus características quedan estructurados en las Tablas 1 y 2, donde se especifican: Autor(es), patología(s), criterios de inclusión/exclusión, seguimiento (Tabla 1), grupo de intervención (GI), grupo control (GC), y resultados (Tabla 2).

En los 7 artículos incluidos en RS existe heterogeneidad, en términos generales, en cuanto al tamaño de la muestra, dado que se encuentran trabajos que oscilan desde los 20 hasta los 96 sujetos, aunque a su vez, más detalladamente, cuatro de los siete artículos presentan homogeneidad en el tamaño, con muestras entre 84-96 sujetos. En cuanto a edad, los trabajos son bastantes homogéneos, oscilando entre los 24,4 y los 31 años.

La enfermedad sobre la que se buscaba incidir era la depresión, por lo que todos los artículos incluidos tenían como tema principal la depresión, que fue evaluada mediante: Quick Inventory of Depressive Symptomatology (Inventario de sintomatología depresiva) (QIDS) (Uebelacker, Battle, Sutton, Magee, \& Miller, 2016), Hospital Anxiety and Depression Scale (Escala hospitalaria de ansiedad y depresión) (HADS) (Satyapriya et al., 2013), Edinburgh Postnatal Depression Scale (Escala de depresión postnatal de Edimburgo) (EPDS) (Davis et al., 2015; Newham, Wittkowski, Hurley, Aplin, \& Westwood, 2014), Structured Clinical interview for Depression (Entrevista clínica estructurada para la depresión) (SCID) (Field, Diego, Delgado, \& Medina, 2013a; Field, Diego, Delgado, \& Medina, 2013b; Field et al., 2012).

Asimismo, los artículos definitivamente incluidos, además de la depresión, buscaban efectos en otras enfermedades como la ansiedad ( 5 estudios), la prematuridad ( 1 estudio) y los trastornos del sueño (1 estudio).

Los estudios llevan a cabo un seguimiento de la asistencia al programa, puesto que indican cuantos sujetos quedan excluidos de los análisis y resultados debido a motivos que les impiden llevar a cabo la intervención de manera continua. 
Tabla 1. Características de los artículos incluidos en la RS: información metodológica (1).

\begin{tabular}{|c|c|c|c|c|c|}
\hline Autor(s)/año & Patología & Muestra & Criterios de inclusión & Criterios de exclusión & Seguimiento \\
\hline $\begin{array}{l}\text { Uebelacker et } \\
\text { al., } 2016\end{array}$ & Depresión prenatal & $\begin{array}{l}20 \text { sujetos } \\
\text { (GC: } 8 ; \mathrm{GI}: 12 \text { ) } \\
\text { (Edad- } \\
\text { GC: } 28,9 \pm 6,0 \text {; } \\
\text { GI: } 28,0 \pm 5,9 \text { ) }\end{array}$ & $\begin{array}{l}\text { 1.Edad gestacional entre } 12-26 \\
\text { semanas } \\
\text { 2.Menor o mayor depresión durante } \\
\text { el embarazo actual } \\
\text { 3.Sintomas depresivos moderados } \\
\text { según el QIDS, puntuación de }>7 \text { y } \\
<20 \\
\text { 4.Médicamente autorizadas a } \\
\text { realizar ejercicio físico moderado; } \\
\text { actualmente no practicantes de yoga } \\
\text { 5.18 años o mayor } \\
\text { 6.Habla inglesa } \\
\text { 7.Disponibilidad para asistir a uno de } \\
\text { los dos horarios de clases }\end{array}$ & $\begin{array}{l}\text { 1.Padecer trastorno bipolar, esquizofrenia, } \\
\text { síntomas psicóticos actuales o crónicos, } \\
\text { TEPT severo, TOC, Trastorno de pánico, } \\
\text { uso peligroso de alcohol o drogas, suicidio } \\
\text { agudo }\end{array}$ & 9 sem \\
\hline $\begin{array}{l}\text { Davis et al., } \\
2015\end{array}$ & $\begin{array}{l}\text { Depresión } \\
\text { Ansiedad materna }\end{array}$ & $\begin{array}{l}46 \text { sujetos } \\
\text { (GC: } 23 ; \mathrm{GI}: 23) \\
\text { (Edad- GC: } \\
30,57 \pm 4,46 ; \mathrm{GI}: \\
29,74 \pm 5,40)\end{array}$ & $\begin{array}{l}\text { 1.Periodo de gestación } \leq 28 \text { semanas } \\
\text { 2.Entre } 18-45 \text { años de edad } \\
\text { 3.Puntuación } \geq 9 \text { en EPDS, } \\
\text { puntuación } \geq 25 \text { en STAI y/o } \\
\text { puntuación } \geq 35 \text { en STAI-T } \\
\text { 4.Realización de } \leq 60 \text { minutos de AF } \\
\text { contemplativa, como yoga o artes } \\
\text { marciales, por semana } \\
\text { 5.Habla inglesa } \\
\text { 6.Disponible para clases de yoga } \\
\text { semanales }\end{array}$ & $\begin{array}{l}\text { 1.Diagnóstico de por vida de esquizofrenia } \\
\text { o trastorno esquizoafectivo, trastorno } \\
\text { bipolar, estado actual de psicosis, trastorno } \\
\text { mental orgánico, retraso generalizado del } \\
\text { desarrollo o cualquier otro trastorno de los } \\
\text { ejes } 1 \text { y } 2 \text { que requiriese de tratamiento } \\
\text { prioritario } \\
\text { 2.Riesgo de suicidio u homicidio } \\
\text { inminente, basado en la entrevista previa } \\
\text { 3. Embarazo indicado medicamente de } \\
\text { alto riesgo, por presencia de condiciones } \\
\text { médicas crónicas o agudas }\end{array}$ & $8 \mathrm{sem}$ \\
\hline
\end{tabular}


Tabla 1. Características de los artículos incluidos en la RS: información metodológica (2).

\begin{tabular}{|c|c|c|c|c|c|}
\hline Autor(es)/año & Patología & Muestra & Criterios de inclusión & Criterios de exclusión & Seguimiento \\
\hline $\begin{array}{l}\text { Newham et } \\
\text { al., } 2014\end{array}$ & $\begin{array}{l}\text { Depresión } \\
\text { Ansiedad materna }\end{array}$ & $\begin{array}{l}59 \text { sujetos } \\
\text { (GC:28; GI:31) } \\
\text { (Edad- GC:31 } \pm 7 \text {; GI: } \\
31 \pm 5)\end{array}$ & $\begin{array}{l}\text { 1.Mujeres sanas }>18 \text { años } \\
2.2^{\circ} \text { o principios del } 3^{\text {er }} \text { trimestre del } \\
\text { embarazo } \\
\text { 3.Primer embarazo } \\
\text { 4.Embarazo sin complicaciones }\end{array}$ & $\begin{array}{l}\text { 1. Mujeres con enfermedad médica, } \\
\text { actualmente en proceso de medicación, o } \\
\text { practicantes de yoga prenatal. }\end{array}$ & $8 \mathrm{sem}$ \\
\hline $\begin{array}{l}\text { Satyapriya et } \\
\text { al., } 2013\end{array}$ & $\begin{array}{l}\text { Depresión } \\
\text { Ansiedad materna }\end{array}$ & $\begin{array}{l}96 \text { sujetos } \\
\text { (GC: } 45 ; \text { GI: } 51 \text { ) } \\
\text { (Edad- } \\
\text { GC: } 24,96 \pm 2,58 ; \text { GI: } \\
\text { 26,41 } \pm 3,01 \text { ) }\end{array}$ & $\begin{array}{l}\text { 1.Edad gestacional entre } 18-20 \\
\text { semanas } \\
\text { 2.Primer embarazo } \\
\text { 3.Multiple embarazo con al menos un } \\
\text { hijo vivo }\end{array}$ & $\begin{array}{l}\text { 1. Problemas médicos asociados (diabetes, } \\
\text { hipertensión, etc.) } \\
\text { 2.Embarazo múltiple } \\
\text { 3.IVF (FIV) } \\
\text { 4.IUGR (RCI) } \\
\text { 5.Anormalidades físico maternales } \\
\text { 6.Anormalidad fetal en la ecografía } \\
\text { 7.Exposición previa al yoga }\end{array}$ & $16 \mathrm{sem}$ \\
\hline $\begin{array}{l}\text { Field et al., } \\
2013 b\end{array}$ & $\begin{array}{l}\text { Depresión prenatal } \\
\text { Ansiedad }\end{array}$ & $\begin{array}{l}92 \text { mujeres } \\
\text { (GC: } 46 ; \text { GI: } 46 \text { ) } \\
\text { (Edad- GC: } 24,5 \pm \\
5,02 ; \text { GI:24,4 } \pm 4,7 \text { ) }\end{array}$ & $\begin{array}{l}\text { 1. Depresión diagnosticada según } \\
\text { SCID } \\
\text { 2.Estar embarazada de un niño }\end{array}$ & $\begin{array}{l}\text { 1.Otras complicaciones de condición } \\
\text { psiquiátrica } \\
\text { 2.Enfermedad médica en el embarazo } \\
\text { 3.Entre } 20-40 \text { años, ambos inclusive } \\
\text { 4.Uso de drogas ilegales }\end{array}$ & $12 \mathrm{sem}$ \\
\hline
\end{tabular}


Tabla 1. Características de los artículos incluidos en la RS: información metodológica (3).

\begin{tabular}{|c|c|c|c|c|c|}
\hline Autor(es)/año & Patología & Muestra & Criterios de inclusión & Criterios de exclusión & Seguimiento \\
\hline $\begin{array}{l}\text { Field et al, } \\
2013 \mathrm{a}\end{array}$ & $\begin{array}{l}\text { Depresión prenatal } \\
\text { Ansiedad } \\
\text { Trastornos del } \\
\text { sueño }\end{array}$ & $\begin{array}{l}92 \text { sujetos } \\
\text { (GC: } 46 \text {; GI: } 46) \\
\text { (Edad- GC: } 26,0 \pm \\
5,6 \text { GI: } 24,4 \pm 4,7 \text { ) }\end{array}$ & $\begin{array}{l}\text { 1. Depresión diagnosticada según SCID } \\
\text { 2.Estar embarazada de un niño } \\
\text { 3.Embarazo sin complicaciones ni enfermedad } \\
\text { médica } \\
\text { 4. }<40 \text { años } \\
\text { 5.No consumir drogas (ni prescritas, ni } \\
\text { ilegales) }\end{array}$ & $\begin{array}{l}\text { 1.No cumplir los criterios de } \\
\text { inclusión }\end{array}$ & $12 \mathrm{sem}$ \\
\hline $\begin{array}{l}\text { Field et al, } \\
2012\end{array}$ & $\begin{array}{l}\text { Depresión prenatal } \\
\text { Prematuridad }\end{array}$ & $\begin{array}{l}84 \text { sujetos } \\
\text { (67 completaron } \\
\text { estudio, GC: } 21 ; \mathrm{GI} \\
\text { Yoga: } 22 ; \text { GI } \\
\text { T.Masaje:24) } \\
\text { (Edad promedio- } \\
26,6 \text { ) }\end{array}$ & $\begin{array}{l}\text { 1. }>18 \text { años de edad } \\
\text { 2.Embarazo de Singleton } \\
\text { 3.Embarazo sin complicaciones } \\
\text { 4.Depresión diagnosticada según SCID }\end{array}$ & $\begin{array}{l}\text { 1Enfermedad médica diagnosticada } \\
\text { (Diabetes, VIH, etc.) } \\
\text { 2. } \geq 40 \text { años } \\
\text { 3.Otra condición psiquiátrica (Ej.: } \\
\text { Trastorno bipolar) } \\
\text { 4.Uso de drogas o medicamentos } \\
\text { automedicados }\end{array}$ & 12 sem. \\
\hline
\end{tabular}

RS: revisión sistemática; GC: Grupo control; GI: Grupo intervención; QIDS: Quick Inventory of Depressive Symptomatology (Inventario de sintomatología depresiva); EPDS: Edinburgh Postnatal Depression Scale (Escala de depresión postnatal de Edimburgo); STAI: State-Trait Anxiety Inventory (Cuestionario de Ansiedad Estado-Rasgo); STAI-T: State Trait Anxiety Inventory Trait Subscale (Subescala del rasgo del cuestionario de Ansiedad Estado-Rasgo); AF: Actividad Física; SCID: Structured Clinical interview for Depression (Entrevista clínica estructurada para la depresión); TEPT: Trastorno de estrés postraumático; TOC: Trastorno obsesivo-compulsivo; IVF (FIV): In Vitro Fertilization (Fecundación in vitro); IUGR (RCI): Intrauterine growth restriction (Retraso del crecimiento intrauterino). 
Tabla 2. Características de los artículos incluidos en la RS: intervención y resultados (1).

\begin{tabular}{|c|c|c|c|}
\hline Autor(es)/año & GI & GC & Resultados \\
\hline $\begin{array}{l}\text { Uebelacker et al., } \\
2016\end{array}$ & $\begin{array}{l}\text { Yoga prenatal } \\
\text { Sesión: } \\
\text { Duración: } 75 \text { min. } \\
\text { Yoga suave } \\
\text { Ejercicios de respiración o meditación } \\
\text { Calentamiento suave } \\
\text { Posturas de pie } \\
\text { Posturas en el suelo } \\
\text { Postura de descanso } \\
\text { Cierre y discusión de la tarea }\end{array}$ & $\begin{array}{l}\text { Educación de salud prenatal: Taller de } \\
\text { bienestar para madres y bebés }\end{array}$ & $\begin{array}{l}\text { No hubo diferencias significativas entre ambos } \\
\text { grupos, aunque hubo mejores resultados en el GI }\end{array}$ \\
\hline Davis et al., 2015 & $\begin{array}{l}\text { Yoga }(1 \mathrm{~d} / \mathrm{sem} .) \\
\text { Sistema tradicional de Ashtanga Vinyasa yoga } \\
\text { modificado para embarazadas } \\
\text { Sesión: } \\
\text { Duración: } 75 \text { min } \\
5 \text { min de introducción a la práctica de la } \\
\text { respiración } \\
10 \text { min sincronización de la respiración: mirada y } \\
\text { movimiento } \\
20 \text { min posturas de pie sincronizadas } \\
20 \text { min posturas sedestación sincronizadas } \\
20 \text { min de enfriamiento en sedestación }\end{array}$ & Tratamiento usual (TAU) & $\begin{array}{l}\text { No se encontraron diferencias significativas } \\
\text { entre ambos grupos en los resultados de } \\
\text { depresión o ansiedad }\end{array}$ \\
\hline
\end{tabular}


Tabla 2. Características de los artículos incluidos en la RS: intervención y resultados (2).

\begin{tabular}{|c|c|c|c|}
\hline Autor(es)/año & GI & GC & Resultados \\
\hline $\begin{array}{l}\text { Newham et al., } \\
2014\end{array}$ & $\begin{array}{l}\text { Yoga }(1 \mathrm{~d} / \mathrm{sem}) \\
\text { Hatha yoga } \\
\text { Sesión: } \\
\text { Ejercicios, posturas y técnicas de relajación } \\
\text { Aplicación del yoga para ayudar dolencias comunes del } \\
\text { embarazo (Sesiones 1-3: posturas para aliviar dolores y } \\
\text { molestias localizadas) } \\
\text { Posición optima del feto (Sesiones 4-5: posiciones } \\
\text { supinas de relajación, ejercicios de fortalecimiento de } \\
\text { espalda y piernas) } \\
\text { Diferentes etapas del parto (Sesiones 6-7) } \\
\text { Periodo posnatal (Sesión 8) }\end{array}$ & Tratamiento usual (TAU) & $\begin{array}{l}\text { No se encontraron diferencias } \\
\text { significativas entre ambos grupos en } \\
\text { los resultados de depresión o ansiedad }\end{array}$ \\
\hline $\begin{array}{l}\text { Satyapriya et al., } \\
2013\end{array}$ & $\begin{array}{l}\text { Yoga integral } \\
\text { Prácticas de yoga }(2 \mathrm{~h} / \mathrm{d}-3 \mathrm{~d} / \mathrm{sem})-4 \mathrm{sem} \\
\text { Práctica de yoga }(1 \mathrm{~h} / \mathrm{d}-7 \mathrm{~d} / \mathrm{sem})-12 \mathrm{sem} \\
\text { Clases de repaso }(2 \mathrm{~h}-1 \text { vez en } 3 \text { semanas hasta la } \\
\text { semana } 28 \text { de gestación y cada } 2 \text { semanas hasta la } \\
\text { semana } 36 \text { de gestación) }\end{array}$ & $\begin{array}{l}\text { Ejercicios prenatales estándar } \\
\text { Prácticas de yoga ( } 2 \mathrm{~h} / \mathrm{d}-3 \mathrm{~d} / \mathrm{sem})-4 \mathrm{sem}\end{array}$ & $\begin{array}{l}\text { Se encontraron diferencias entre los } \\
\text { grupos GI y GC tanto en la ansiedad } \\
\text { como en la depresión }\end{array}$ \\
\hline
\end{tabular}


Tabla 2. Características de los artículos incluidos en la RS: intervención y resultados (3).

\begin{tabular}{|c|c|c|c|c|}
\hline Autor(es)/año & \multicolumn{2}{|l|}{ Grupo de intervención } & Grupo control & Resultados \\
\hline Field et al., 2013b & \multicolumn{2}{|c|}{$\begin{array}{l}\text { Yoga }(1 \mathrm{~d} / \mathrm{sem}) \\
\text { Sesiones cortas de } 20 \mathrm{~min} \\
\text { Rutina adaptada a mujeres en su segundo y tercer } \\
\text { trimestre de gestación }\end{array}$} & Clases de apoyo social & $\begin{array}{l}\text { El grupo de yoga mostró un cambio } \\
\text { significativo pretest, postest en las } \\
\text { sesiones con respecto al grupo control }\end{array}$ \\
\hline Field et al., 2013a & \multicolumn{2}{|c|}{$\begin{array}{l}\text { Yoga/Tai chi }(1 \mathrm{~d} / \mathrm{sem}) \\
\text { Sesión: } \\
\text { Duración: } 20 \text { min } \\
\text { Rutina adaptada a mujeres en su segundo y tercer } \\
\text { trimestre de gestación }\end{array}$} & Lista de espera & $\begin{array}{l}\text { El grupo de yoga/Tai chi mostró } \\
\text { mejores resultados con respecto a todas } \\
\text { las variables de estudio }\end{array}$ \\
\hline Field et al., 2012 & $\begin{array}{l}\text { GI 1: Yoga } \\
\text { Sesión: } \\
\text { Duración: } 20 \text { min } \\
\text { Rutina adaptada a mujeres } \\
\text { en su segundo y tercer } \\
\text { trimestre de gestación }\end{array}$ & $\begin{array}{l}\text { GI 2: Terapia de masaje } \\
\text { Sesión: } \\
\text { Duración: } 20 \text { min } \\
\text { Cabeza, espalda, piernas y } \\
\text { brazos }\end{array}$ & Clases de atención prenatal estándar & $\begin{array}{l}\text { Tanto el GI1 como el GI2 obtuvieron } \\
\text { buenos y semejantes resultados con } \\
\text { respecto al GC }\end{array}$ \\
\hline
\end{tabular}

RS: revisión sistemática; GI: Grupo de intervención; GI1: Grupo de intervención 1; GI2: Grupo de intervención 2; GC: Grupo control; h: hora; min: minutos; d: días; sem: semana. 


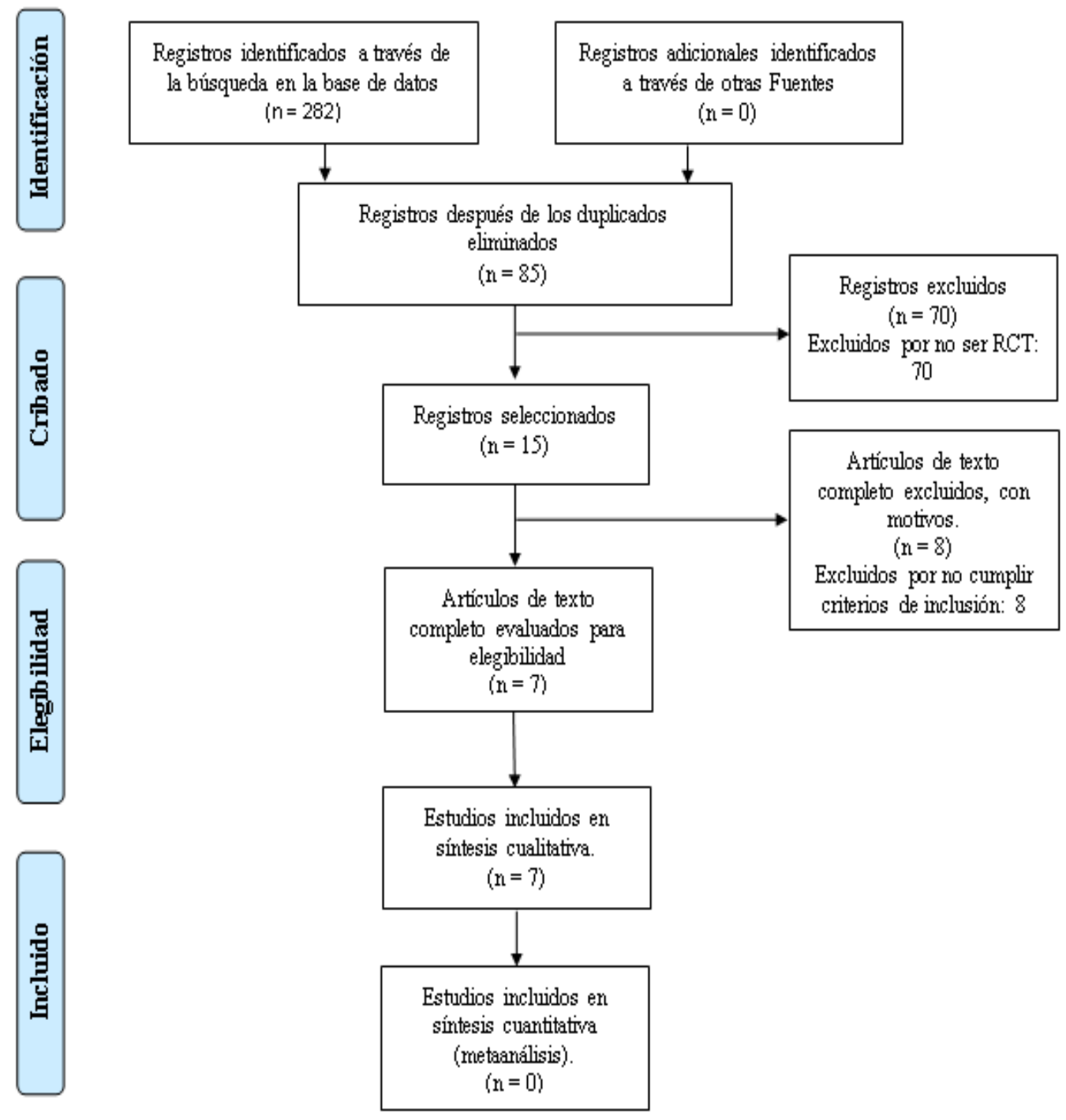

Figura 1. Diagrama de flujo de las fases de cribado de la revisión sistemática.

Con respecto a la frecuencia semanal con la que se llevó a cabo la intervención, la mayoría de los estudios optó por una sesión semanal (6 estudios), mientras que el estudio restante lo hizo por una sesión diaria.

En lo referido a la duración de las sesiones, la mayoría de los estudios (4 estudios) se decantó por sesiones largas ( $>1$ hora), mientras que los restantes (3 estudios) realizaron sesiones cortas de 20 min.

En cuanto a la fase de seguimiento la mayoría (4 estudios) realizó un seguimiento de 12 semanas o superior, mientras que el de los restantes (3 artículos) fue de 8-9 semanas. Este aspecto puede conllevar diferencias en los resultados.

Las modalidades de Yoga que llevaron a cabo los estudios fueron: Yoga integral (Satyapriya et al., 2013), Hatha Yoga (Newham et al., 2014), Ashtanga Vinyasa Yoga (Davis et al., 2015), Yoga prenatal (Uebelacker et al., 2016) y Yoga adaptado al segundo y tercer trimestre de embarazo (Field et al., 2013a; Field et al., 2013b; Field et al., 2012). En cambio, 
todos ellos realizaron alguna adaptación de la modalidad de Yoga debido al embarazo, predominando en las sesiones una intensidad leve-moderada. Además, las sesiones estaban compuestas por respiración (Pranayama), posturas físicas (Asanas) y meditación/relajación (Davis et al., 2015; Newham et al., 2014; Satyapriya et al., 2013; Uebelacker et al., 2016). En el caso de Field et al. (2013a, 2013b) y Field et al. (2012) solo se llevaron a cabo Asanas.

\section{Resultados individuales de los estudios}

Los resultados de los artículos seleccionados en la RS fueron los siguientes y, además, pueden consultarse de manera detallada en la Tabla 2.

Uebelacker et al. (2016) estudiaron el efecto del Yoga en mujeres en periodo de gestación que se encontrasen en un proceso depresivo. Al finalizar el estudio y realizar las evaluaciones pertinentes, se obtuvo una reducción de la depresión que padecían las gestantes al inicio del programa.

Davis et al. (2015) llevaron a cabo un estudio cuyo propósito era comprobar los efectos del Yoga en mujeres gestantes con depresión y ansiedad. Los resultados obtenidos fueron favorables a nivel intra-grupo, pero la diferencia entre grupos no fue concluyente.

En otro estudio, Newham et al. (2014) querían comprobar los efectos que el Yoga podía provocar en mujeres con depresión y ansiedad en plena situación de embarazo. La depresión se redujo tanto en el GI $(p<0,0001)$, como en el GC $(p=0,04)$ observando diferencias significativas $(\mathrm{p}<0,05)$.

Por su lado, Satyapriya et al. (2013) estudiaron los efectos del Yoga sobre la depresión y la ansiedad que padecían mujeres gestantes. La depresión se redujo de forma significativa en el GI $(p<0,001)$. Además, se encontraron diferencias significativas entre el GI y el GC $(p<0,001)$.

Field et al. (2013b) basaron su trabajo en comprobar si resultaba más efectivo para tratar la ansiedad y la depresión prenatal someter a mujeres embarazas a sesiones de Yoga o a clases de apoyo social. Al terminar su estudio, los resultados fueron favorables con respecto al grupo de Yoga, siendo resultados significativos $(\mathrm{p}<0,01)$.

En otro trabajo, Field et al. (2013a) estudiaron los efectos que el Yoga/Tai Chi, trabajados conjuntamente por un mismo grupo, podía producir en las variables: depresión prenatal, ansiedad, y trastornos del sueño, que sufrían mujeres en periodo de gestación. Al finalizar el periodo de seguimiento, obtuvieron resultados favorables en el grupo Yoga/Tai Chi $(\mathrm{p}<0,001)$ donde las variables sometidas a estudio disminuyeron.

A su vez, Field et al. (2012) realizaron un estudio con el propósito de comprobar los efectos que dos intervenciones, una basada en el Yoga y otra en terapias de masaje, podían lograr en la depresión prenatal y la prematuridad de mujeres en situación de gestación. Tras finalizar el estudio, los dos grupos de intervención obtuvieron resultados favorables y similares en cuanto a las variables de estudio $(\mathrm{p}<0,001)$.

\section{Riesgo de sesgo o calidad metodológica de los estudios}

Según Van Tulder et al. (2003), la presente RS estaría clasificada como de evidencia sólida, puesto que los 7 artículos que la componen son considerados de alta calidad metodológica según la escala PEDro. Estos resultados pueden consultarse en la Tabla 3. 
Tabla 3. Evaluación de la calidad metodológica de cada artículo según escala PEDro.

\begin{tabular}{|c|c|c|c|c|c|c|c|c|c|c|c|c|}
\hline Publicación & criterio 1 & criterio 2 & criterio 3 & criterio 4 & criterio 5 & criterio 6 & criterio 7 & criterio 8 & criterio 9 & criterio 10 & criterio 11 & Total \\
\hline $\begin{array}{l}\text { Uebelacker } \\
\text { et al., } 2016\end{array}$ & 1 & 0 & 0 & 1 & 0 & 0 & 0 & 1 & 1 & 1 & 1 & 6 \\
\hline $\begin{array}{l}\text { Davis et al., } \\
\quad 2015\end{array}$ & 1 & 1 & 1 & 1 & 1 & 1 & 0 & 1 & 1 & 1 & 1 & 10 \\
\hline $\begin{array}{l}\text { Newham et } \\
\text { al., } 2014\end{array}$ & 1 & 1 & 1 & 1 & 0 & 1 & 0 & 1 & 1 & 1 & 1 & 9 \\
\hline $\begin{array}{l}\text { Satyapriya et } \\
\text { al., } 2013\end{array}$ & 1 & 1 & 1 & 1 & 0 & 0 & 1 & 1 & 1 & 1 & 1 & 9 \\
\hline $\begin{array}{l}\text { Field et al., } \\
2013 \text { (b) }\end{array}$ & 1 & 1 & 1 & 1 & 0 & 0 & 1 & 1 & 1 & 1 & 1 & 9 \\
\hline $\begin{array}{l}\text { Field et al., } \\
2013 \text { (a) }\end{array}$ & 1 & 1 & 0 & 1 & 0 & 0 & 0 & 1 & 1 & 1 & 1 & 7 \\
\hline $\begin{array}{c}\text { Field et al., } \\
2012\end{array}$ & 1 & 1 & 0 & 1 & 0 & 0 & 0 & 1 & 1 & 1 & 1 & 7 \\
\hline
\end{tabular}

Criterio 1: Criterio de elegibilidad; Criterio 2: Aleatorización; Criterio 3: Aleatorización oculta; Criterio 4: Similitud basal entre grupos; Criterio 5: Cegamiento de los sujetos; Criterio 6: Cegamiento de terapeutas; Criterio 7: Cegamiento de evaluadores; Criterio 8: Más del 85\% de los resultados de los sujetos se midieron; Criterio 9: Intención de tratar; Criterio 10: Comparación entre grupos; Criterio 11: Se utilizan medidas puntuales y de variabilidad. 


\section{Discusión}

Los estudios RCT de la presente RS tienen una variabilidad amplia en cuanto al rango de validez interna, encontrando valoraciones de 6 hasta 10 puntos, donde predominan los artículos con valoraciones altas ( 9 puntos).

El artículo de Davis et al. (2015), que es el mejor valorado de esta RS, no refleja diferencias significativas en la reducción de los índices de depresión en su estudio, que comparó el Yoga prenatal con clases de atención prenatal. Además, los estudios de Uebelacker et al. (2016) y Newham et al. (2014), cuyas valoraciones son 6 y 9 puntos respectivamente, compararon la práctica de Yoga prenatal con clases de atención prenatal, no encontrando diferencias entre ambos en cuanto a resultados se refiere. Los tres estudios anteriores no obtuvieron los resultados esperados, debido a que la mejoría producida por el programa con Yoga estaba equiparada a la conseguida en las clases de atención prenatal, donde algunas razones pueden ser el periodo de seguimiento ( $\leq 9$ semanas) y al número de sujetos que participaron ( $<60$ sujetos). El tamaño muestral puede determinar en gran medida la obtención de la suficiente evidencia para rechazar o no la hipótesis nula (Rondón \& Rodríguez, 2007), por lo que se considera un factor relevante a la hora de encontrar resultados significativos en los estudios revisados. Por otra parte, las sesiones eran de duración larga ( $>1$ hora) y la calidad metodológica alta ( $\geq 6$ puntos).

Por otro lado, Satyapriya et al. (2013), Field et al. (2013b), y Field et al. (2013a), en sus respectivos trabajos obtuvieron que en los diferentes GI, basados en Yoga integrado (Satyapriya et al., 2013), Yoga (Field et al., 2013b), y Yoga/Tai Chi (Field et al., 2013a), los resultados respecto a los índices de depresión reflejaron ser mejores que los obtenidos en el GC, donde los sujetos recibían clases de apoyo social (Field et al., 2013b), ejercicios prenatales estándar (Satyapriya et al., 2013), y lista de espera (Field et al., 2013a). Esto puede deberse al número de sujetos que participó en el estudio ( $>90$ sujetos), a un periodo de seguimiento alto ( $\geq 12$ semanas), y a una alta calidad metodológica ( $\geq 7$ puntos). En cuanto a la duración de las sesiones, Satyapriya et al. (2013) llevó a cabo sesiones largas ( $>1$ hora), mientras que los estudios de Field et al. (2013a, 2013b) realizaron sesiones cortas ( $<1$ hora). Siguiendo los resultados de estos tres últimos estudios, la duración de las sesiones no es determinante a la hora de obtener mejoras en los índices de depresión de mujeres gestantes con depresión, por lo que se pueden recomendar sesiones de más de 1 hora o de menos de 1 hora.

Por último, Field et al. (2012) realizaron la comparación de tres grupos: dos GI, uno de ellos practicaba Yoga y el otro recibía terapias de masaje, y un GC, que recibía clases de atención prenatal estándar. Los resultados en los GI fueron mejores que en el GC, donde tanto la práctica de Yoga, como las terapias de masaje consiguieron resultados similares. El hecho de realizar tres comparaciones posibilita una mayor variedad de resultados y, por tanto, visualizar mejor los efectos ocurridos en dichos grupos. Los buenos resultados de esta investigación se pueden deber tanto al número de sujetos que participó en el estudio ( $>80$ sujetos), como a un periodo de seguimiento de los sujetos de 12 semanas; y a una alta calidad metodológica ( 7 puntos). Por su lado, la duración de las sesiones fue corta $(<1$ hora). 
La diferencia existente en los estudios incluidos en la RS en cuanto a la relación calidad metodológica-resultados se achaca a la duración de seguimiento de los mismos, así como al tamaño muestral.

\section{Limitaciones y futuras líneas de investigación}

La mayor limitación de esta RS es la falta de estudios publicados centrados en la temática en torno a la que gira la misma. Otra limitación del trabajo ha sido la heterogeneidad en los resultados de los diferentes estudios, hecho que no ha permitido una correcta y clara síntesis y conocimiento de los efectos que el Yoga puede producir en mujeres gestantes con depresión. En futuras investigaciones sobre este tema se podría buscar la comparación de un grupo de Yoga con más de un grupo control a la vez, los cuales realicen otro tipo de actividad, para así comprobar en un mismo estudio los beneficios conseguidos en los distintos grupos por actividades diferentes, y así tener más evidencias acerca de qué actividades son más beneficiosas para reducir el índice de depresión en mujeres gestantes.

\section{Conclusiones}

El Yoga como método de trabajo cuerpo-mente produce mejoras en cuanto a los índices de depresión en mujeres gestantes con depresión si se realiza al menos una sesión semanal con una duración mínima de 20 minutos/sesión, y adaptado al embarazo. Además, la intervención debe durar 12 semanas o más y el tamaño muestral debe ser $>60$. La adaptación de la intervención a los cambios físicos producidos por el embarazo parece no influir en los resultados.

\section{Referencias}

Aznal, S. S., Wong, C., Tan, P. L., See, V. V. \& Wong, C. K. (2014). Mother's mental preparedness for pregnancy: The affecting factors and its effect on birth outcomes. Editorial Assistant, 19.

Battle, C., Uebelacker, L., Magee, S., Sutton, K. \& Miller, I. (2015). Potential for prenatal yoga to serve as an intervention to treat depression during pregnancy. Womens Health Issues, 25(2), 134-141. doi:10.1016/j.whi.2014.12.003

Beddoe, A., Yang, C., Kennedy, H., Weiss, S. \& Lee, K. (2009). The effects of mindfulness based yoga during pregnancy on maternal psychological and physical distress. Journal of Obstetric, Gynecologic, and Neonatal Nursing, 38(3), 310-319. doi:10.1111/j.15526909.2009.01023.x

Belay, Y., Moges, N., Hiksa, F., Arado, K. \& Liben, M. (2018). Prevalence of Antenatal Depression and Associated Factors among Pregnant Women Attending Antenatal Care at Dubti Hospital: A Case of Pastoralist Region in Northeast Ethiopia. Depression Research and Treatment, 1-9. doi:10.1155/2018/1659089 
Byatt, N., Simas, T., Lundquist, R. S., Johnson, J. V. \& Ziedonis, D. M. (2012). Strategies for improving perinatal depression treatment in North American outpatient obstetric settings. Journal of Psychosomatic Obstetrics \& Gynecology, 33(4), 143-161. doi:10.3109/0167482X.2012.728649

Chandler, K. (2001). The emerging field of yoga therapy. Hawaii Medical Journal, 60, 286287. doi:https://www.ncbi.nlm.nih.gov/pubmed/11797492

Chida, Y. \& Steptoe, A. (2009). Cortisol awakening response and psychosocial factors: a systematic review and meta-analysis. Biological Psychology, 80(3), 265-278. doi:10.1016/j.biopsycho.2008.10.004

Chuntharapat, S., Petpichetchian, W. \& Hatthakit, U. (2008). Yoga during pregnancy: effects on maternal comfort, labor pain and birth outcomes. Complementary Therapies in Clinical Practice, 14(2), 105-115. doi:10.1016/j.ctcp.2007.12.007

Davis, K., Goodman, S., Leiferman, J., Taylor, M. \& Dimidjian, S. (2015). A randomized controlled trial of yoga for pregnant women with symptoms of depression and anxiety. Complementary Therapies in Clinical Practice, 21(3), 166-172. doi:10.1016/j.ctcp.2015.06.005

de Bruijn, A., van Bakel, H. \& van Baar, A. (2009). Sex differences in the relation between prenatal maternal emotional complaints and child outcome. Early human development, 85, 319-324. doi:10.1016/j.earlhumdev.2008.12.009

Deave, T., Heronm, J., Evans, J. \& Edmond, A. (2008). The impact of maternal depression in pregnancy on early child development. BJOG: An International Journal of Obstetrics \& Gynaecology, 115, 1043-1051. doi:10.1111/j.1471-0528.2008.01752.x

Einarson, A., Choi, J., Einarson, T. \& Koren, G. (2010). Adverse effects of antidepressant use in pregnancy: an evaluation of fetal growth and preterm birth. Depression and Anxiety, 27(1), 35-38. doi:10.1002/da.20598

Elkins, M., Herbert, R., Moseley, A., Sherrington, C. \& Maher, C. (2010). Rating the quality of trials in systematic reviews of physical therapy interventions. Cardiopulmonary Physical Therapy Journal, 20-26. doi:https://www.ncbi.nlm.nih.gov/pmc/articles/PMC2941354/

Enkin, M., Keirse, M., Neilson, J., Crowther, C., Duley, L., Hodnett, E. \& Hofmeyr, J. (2000). A Guide to Effective Care in Pregnancy and Childbirth. Oxford University Press. doi:10.1093 / med / 9780192631732.001.0001

Field, T., Diego, M., Delgado, J. \& Medina, L. (2013a). Tai chi/yoga reduces prenatal depression, anxiety and sleep disturbances. Complementary Therapies in Clinical Practice, 19(1), 6-10. doi:10.1016/j.ctcp.2012.10.001

Field, T., Diego, M., Delgado, J. \& Medina, L. (2013b). Yoga and social support reduce prenatal depression, anxiety and cortisol. Journal of Bodywork and Movement Therapies, 17(4), 397-403. doi:10.1016/j.jbmt.2013.03.010

Field, T., Diego, M., Hernandez-Reif, M., Deeds, O., Holder, V., Schanberg, S. \& Kuhn, C. (2009). Depressed pregnant black women have a greater incidence of prematurity and low birthweight outcomes. Infant Behavior and Development, 32, 10-16. doi:10.1016/j.infbeh.2008.09.005 
Field, T., Diego, M., Hernandez-Reif, M., Medina, L., Delgado, J. \& Hernandez, A. (2012). Yoga and massage therapy reduce prenatal depression and prematurity. Journal of Bodywork and Movement Therapies, 16(2), 204-209. doi:10.1016/j.jbmt.2011.08.002

Gómez-Conesa, A. (30 de Diciembre de 2012). PEDro. Physiotherapy evidence database. Recuperado el 13 de Marzo de 2019, de https://www.pedro.org.au/spanish/downloads/pedro-scale/

Goodman, J. (2009). Women's Attitudes, Preferences, and Perceived Barriers to Treatment for Perinatal Depression. Birth Issues in Perinatal Care, 36(1), 60-69. doi:10.1111/j.1523536X.2008.00296.X

Goodman, S. \& Tully, E. (2009). Recurrence of depression during pregnancy: psychosocial and personal functioning correlates. Depression \& Anxiety, 26(6), 557-567. doi:10.1002/da.20421

Hay, D., Pawlby, S., Waters, C., Perra, O. \& Sharp, D. (2010). Mothers' antenatal depression and their children's antisocial outcome. Child Development, 81, 149-165. doi:10.1111/j.1467-8624.2009.01386.x

Heron, J., O'Connor, T., Evans, J., Golding, J., Glover, V. \& Team, T. A. (2004). The course of anxiety and depression through pregnancy and the postpartum in a community sample. Journal of Affective Disorders, 80(1), 65-73. doi:10.1016/j.jad.2003.08.004

Mian, A. (2005). Depression in pregnancy and the postpartum period: balancing adverse effects of untreated illness with treatment risks. Journal of Psychiatric Practice, 11(6), 389396. doi:https://www.ncbi.nlm.nih.gov/pubmed/16304507

Misri, S., Kendrick, K., Oberlander, T., Norris, S., Tomfohr, L., Zhang, H. \& Grunau, R. (2010). Antenatal depression and anxiety affect postpartum parenting stress: A longitudinal, prospective study. Canadian Journal of Psychiatry, 55(4), 222-228. doi:10.1177\%2F070674371005500405

Mulder, E., de Medina, P., Huizink, A., Van den Bergh, B., Buitelaar, J. \& Visser, G. (2002). Prenatal maternal stress: effects on pregnancy and the unborn child. Early Human Development, 70, 3-14. doi:10.1016/S0378-3782(02)00075-0

Newham, J., Wittkowski, A., Hurley, J., Aplin, J. \& Westwood, M. (2014). Effects of antenatal yoga on maternal anxiety and depression: a randomized controlled trial. Depression \& Anxiety, 31(8), 631-640. doi:10.1002/da.22268

Ngai, F. \& Chan, S. (2011). Psychosocial factors and maternal wellbeing: an exploratory path analysis. International Journal of Nursing Studies, 48(6), 725-731. doi:10.1016/j.ijnurstu.2010.11.002

O'Donnell, K., O'Connor, T. \& Glover, V. (2009). Prenatal stress and neurodevelopment of the child: focus on the HPA axis and role of the placenta. Developmental Neuroscience, 31(4), 285-292. doi:10.1159/000216539

Obel, C., Hedegaard, M., Henriksen, T., Secher, N., Olsen, J. \& Levine, S. (2005). Stress and salivary cortisol during pregnancy. Psychoneuroendocrinology, 30(7), 647-656. doi:10.1016/j.psyneuen.2004.11.006

Parker, G., Crawford, J. \& Hadzi-Pavlovic, D. (2008). Quantified superiority of cognitive behavior therapy to antidepressant drugs: a challenge to an earlier meta-analysis. Acta Psychiatrica Scandinavica, 118(2), 91-97. doi:10.1111/j.1600-0447.2008.01196.x 
Rakhshani, A., Nagarathna, R., Mhaskar, R., Mhaskar, A., Thomas, A. \& Gunasheela, S. (2012). The effects of yoga in prevention of pregnancy complications in high-risk pregnancies: a randomized controlled trial. Preventive Medicine, 55(4), 333-340. doi:10.1016/j.ypmed.2012.07.020

Rondón, M. A. \& Rodríguez, V. A. (2007). Algunos conceptos básicos para el cálculo del tamaño de la muestra. Universitas Médica, 48(3), 334-339.

Satyapriya, M., Nagarathna, R., Padmalatha, V. \& Nagendra, H. (2013). Effect of integrated yoga on anxiety, depression \& well being in normal pregnancy. Complementary Therapies in Clinical Practice, 19(4), 230-236. doi:10.1016/j.ctcp.2013.06.003

Sturrock, W. A. \& Johnson, J. A. (1990). The relationship between childbirth education classes and obstetric outcome. BIRTH issues in perinatal care, 17(2), 82-85. doi:10.1111/j.1523-536x.1990.tb00704.x

Sun, Y., Hung, Y., Chang, Y. \& Kuo, S. (2010). Effects of a prenatal yoga programme on the discomforts of pregnancy and maternal childbirth self-efficacy in Taiwan. Midwifery, 26(6), 31-36. doi:10.1016/j.midw.2009.01.005

Szegda, K., Bertone-Johnson, E. R., Pekow, P., Powers, S., Markenson, G., Dole, N. \& Chasan-Taber, L. (2018). Physical activity and depressive symptoms during pregnancy among Latina women: a prospective cohort study. BMC Pregnancy and childbirth, 18(1), 252. doi:10.1186\%2Fs12884-018-1839-5

Uebelacker, L., Battle, C., Sutton, K., Magee, S. \& Miller, I. (2016). A pilot randomized controlled trial comparing prenatal yoga to perinatal health education for antenatal depression. Archives of Women's Mental Health, 19(3), 543-547. doi:10.1007/s00737015-0571-7

Urrutia, G. \& Bonfill, X. (2010). Declaración PRISMA: una propuesta para mejorar la publicación de revisiones sistemáticas y metaanálisis. Medicina Clínica, 135(11), 507511. doi:10.1016/j.medcli.2010.01.015

Van Tulder, M., Furlan, A., Bombardier, C. \& Bouter, L. (2003). Updated Method Guidelines for Systematic Reviews in the Cochrane Collaboration Back Review Group. Spine, 28(12), 1290-1299. doi:10.1097/01.BRS.0000065484.95996.AF

WHO. (Marzo de 2017). Depression and other common mental disorders: global health estimates. Recuperado el 12 de Abril de 2019, de http://iris.paho.org/xmlui/handle/123456789/34006 